\title{
Decomposing electronic and lattice contributions in optical pump-X-ray probe transient inner-shell absorption spectroscopy of CuO
}

\author{
Johannes Mahl ${ }^{1,2}$, Stefan $\mathrm{Nepp}^{1,3}$, Friedrich Roth $^{4,5}$, Catherine Saladrigas ${ }^{1,6}$, Hendrik \\ Bluhm $^{1,7}$ Jinghua Guo ${ }^{7}$, Wanli Yang ${ }^{7}$, Nils Huse ${ }^{2,5}$, Wolfgang Eberhardt ${ }^{5,7}$, and Oliver \\ Gessner $^{1}$ \\ ${ }^{1}$ Chemical Sciences Division, Lawrence Berkeley National Laboratory, Berkeley, CA, 94720 USA \\ ${ }^{2}$ Physics Department Universität Hamburg, 22607 Hamburg, Germany \\ ${ }^{3}$ Helmholtz-Zentrum Berlin für Materialien und Energie, 14109 Berlin, Germany \\ ${ }^{4}$ Institute for Experimental Physics, TU Bergakademie Freiberg, 09599 Germany \\ ${ }^{5}$ Center for Free-Electron Laser Science DESY, 22607 Hamburg, Germany \\ ${ }^{6}$ Chemistry Department UC Berkeley, CA, 94720 USA \\ ${ }^{7}$ Advanced Light Source, Lawrence Berkeley National Laboratory, Berkeley, CA, 94720 USA
}

\begin{abstract}
Electronic and lattice contributions to transient X-ray absorption spectra of $\mathrm{CuO}$ are analyzed using picosecond time-resolved and temperature-dependent measurements. Super-bandgap excitation with $355 \mathrm{~nm}$ and $532 \mathrm{~nm}$ laser pulses leads to significantly different trends.
\end{abstract}

\section{Introduction}

Emerging ultrafast X-ray light source technologies such as X-ray Free Electron Lasers (X-FELs) promise to provide entirely new ways of probing ultrafast electronic and nuclear dynamics in matter. It is crucial to develop a better understanding of the X-ray spectroscopic fingerprints of various excitations and their interactions. Electron-phonon coupling, for example, leads to a time-dependent convolution of electronic and lattice contributions to transient X-ray spectra, which need to be disentangled to understand the underlying physics [1]. Coupled electronic and nuclear dynamics in transition metal oxides are of particular interest for many electronic, chemical, and energy-storage applications [2]. Here, we study photoinduced dynamics in cupric oxide $(\mathrm{CuO})$ by picosecond time-resolved $\mathrm{X}$-ray absorption spectroscopy (tr-XAS) and temperature-dependent quasi-static XAS in order to explore the relative contributions of charge carrier dynamics and lattice heating to transient X-ray spectra of solid samples.

\section{Experimental Setup}

$\mathrm{CuO}$ samples are excited with $10 \mathrm{ps}$ laser pulses of either $532 \mathrm{~nm}(2.33 \mathrm{eV})$ or $355 \mathrm{~nm}$ $(3.5 \mathrm{eV})$ wavelength (photon energy), both of which are above the band gap of $\mathrm{CuO}(\leq 1.9$ 
eV [3]). Tr-XAS signals are recorded using 70 ps FWHM X-ray pulses from the Advanced Light Source (ALS). A time-stamping technique enables simultaneous acquisition of trXAS signals from an extended X-ray pulse train with 2 ns pulse-to-pulse spacing [4]. The tr-XAS results are compared to temperature-dependent, quasi-static X-ray absorption spectra recorded independently. Both experiments are performed in total X-ray fluorescence yield (TFY) mode.

\section{Results and Discussion}

Laser fluences between $4 \mathrm{~mJ} / \mathrm{cm}^{2}$ and $15 \mathrm{~mJ} / \mathrm{cm}^{2}$ are employed for excitation at $532 \mathrm{~nm}$ and $355 \mathrm{~nm}$. Some fluences enable a direct comparison between the two tr-XAS data sets, taking into account differences in optical absorption as well as optical and X-ray penetration/escape depths specific to the experimental geometry. They are chosen such that the pump-probe contrast, i.e. the ratio of detected X-ray fluorescence events from excited and ground state sites within the sample should be comparable for the $532 \mathrm{~nm}$ and $355 \mathrm{~nm}$ experiments.
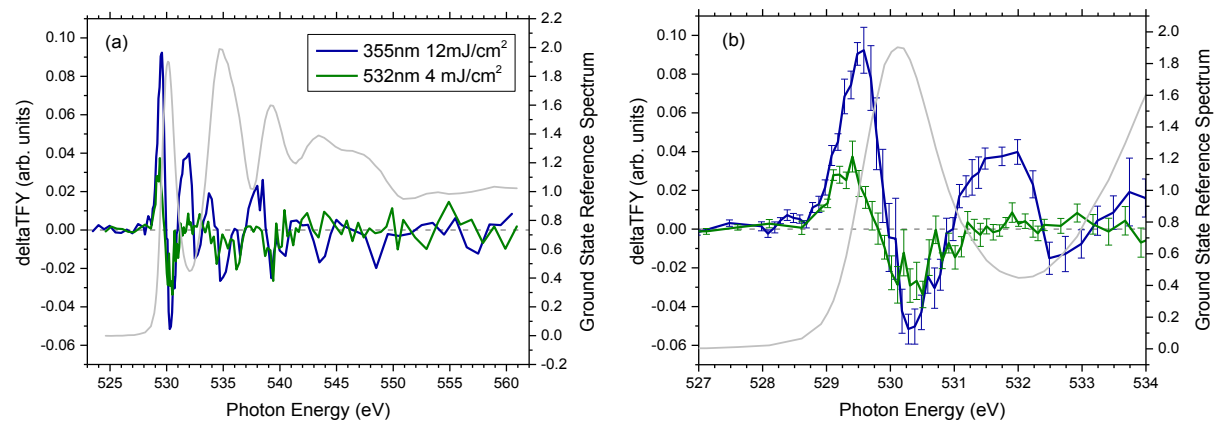

Fig. 1. a) Laser-induced changes in the oxygen $\mathrm{K}$-edge absorption spectrum of $\mathrm{CuO}$ at laser wavelengths of $532 \mathrm{~nm}$ (green) and $355 \mathrm{~nm}$ (blue) compared to the static near-edge absorption spectrum (gray). b) Magnified view of the first resonance at $530.1 \mathrm{eV}$. The right and left axes correspond to the ground state spectra and transient differences, respectively. Error bars indicate standard errors of 10 individual acquisitions.

Figure 1a shows the ground state oxygen $\mathrm{K}$-edge absorption spectrum of $\mathrm{CuO}$ (gray, right $\mathrm{y}$-axis) as well as the differences between ground and excited state spectra for both excitation wavelengths at a pump-probe delay of $150 \mathrm{ps}$ (green, blue, left y-axis). The laserinduced changes in the absorption spectra are most prominent near the lowest energy peak at $530.1 \mathrm{eV}$, which is shown in more detail in Fig. 1b. The change in the tr-XAS spectra is substantially more pronounced for UV excitation compared to visible excitation. The maximal difference between ground and excited state spectra is a factor of 3 larger for UV excitation. Furthermore, an additional difference peak appears near $531.5 \mathrm{eV}$ for the UVexcited sample that is absent for $532 \mathrm{~nm}$ excitation. A detailed analysis of this signal with respect to either thermal effects and/or partial conversion of $\mathrm{CuO}$ to $\mathrm{Cu}_{2} \mathrm{O}$ by the $355 \mathrm{~nm}$ radiation is currently in progress. 

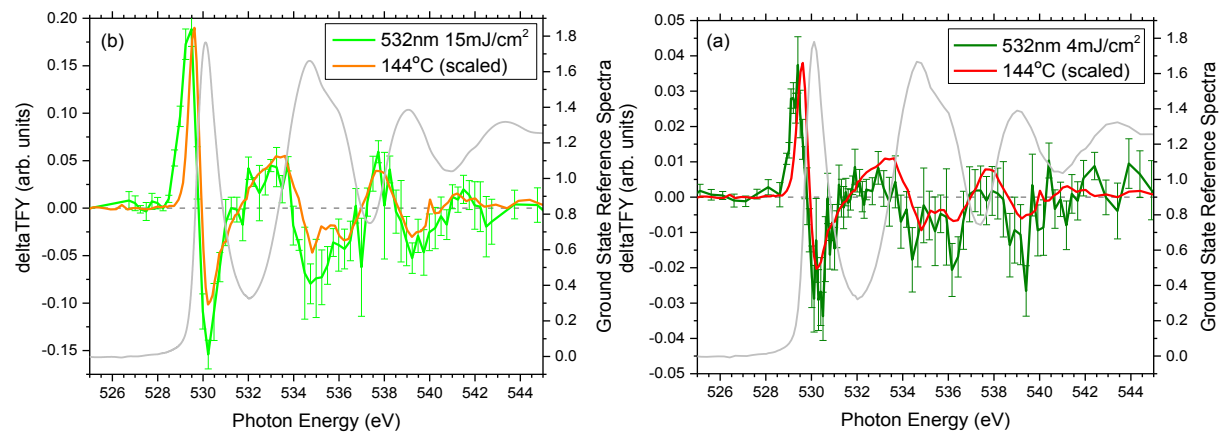

Fig. 2. Photoinduced tr-XAS difference spectra of $\mathrm{CuO}$ (green, left axes) recorded 150 ps after excitation with $532 \mathrm{~nm}$ pulses at intensities of a) $4 \mathrm{~mJ} / \mathrm{cm}^{2}$ and b) $15 \mathrm{~mJ} / \mathrm{cm}^{2}$. The spectra are compared to the difference between quasi-static XAS spectra recorded at room temperature and $144^{\circ} \mathrm{C}$, scaled to the same maxima as the tr-XAS signals (red). The ground state XAS spectrum of $\mathrm{CuO}$ is shown in gray (right axes).

Generally, tr-XAS spectra recorded with $532 \mathrm{~nm}$ excitation are expected to be less affected by thermal effects than their $355 \mathrm{~nm}$ counterparts since ca. $1.2 \mathrm{eV}$ less energy per photon is converted into phonons during intraband relaxation to the conduction band minimum. Figure 2 compares tr-XAS difference spectra (green) after $532 \mathrm{~nm}$ excitation with intensities of a) $4 \mathrm{~mJ} / \mathrm{cm}^{2}$ and b) $15 \mathrm{~mJ} / \mathrm{cm}^{2}$ with temperature-induced, quasi-static difference spectra (red). The differences exhibit obvious similarities, indicating significant contributions of lattice heating to the tr-XAS spectra. However, the laser-induced difference spectra exhibit additional contributions at the low-energy side of the main feature $(\sim 529 \mathrm{eV})$, which become more prominent at higher excitation fluences. A more detailed analysis and theoretical calculations are underway to identify the nature of this feature, in particular, with respect to possible contributions from electronic excitations.

This work was supported by the U.S. Department of Energy, Office of Science, Office of Basic Energy Sciences, Chemical Sciences, Geosciences and Biosciences Division, through Contract No. DE-AC02-05CH11231. JM acknowledges partial support from the ALS Doctoral Fellowship in Residence Program. SN acknowledges support by the Alexander von Humboldt foundation.

\section{References}

1. D. Hayes, R. G. Hadt, J. D. Emery, A. A. Cordones, A. B. F. Martinson, M. L. Shelby, K. A. Fransted, P. D. Dahlberg, J. Hong, X. Zhang, Q. Kong, R. W. Schoenlein, and L. X. Chen, Energy Environ. Sci. 9, 3754 (2016)

2. J.Y. Xiang, J.P. Tu, L. Zhang, Y. Zhou, X.L.Wang, S.J. Shi., J. Power Sources 195, $313(2010)$

3. C. E. Ekuma, V. I. Anisimov, J. Moreno, and M. Jarrell, Eur. Phys. J. B 87, 1 (2014)

4. S. Neppl, J. Mahl, A. S. Tremsin, B. Rude, R. Qiao, W. Yang, J. Guo, and O. Gessner, Faraday Discuss. 194, 659 (2016) 